\title{
Detent Force Analysis in Permanent Magnet Linear Synchronous Motor Considering Longitudinal End Effects
}

\author{
Liyi Li*, Mingna Ma* and C.C.Chan*
}

\begin{abstract}
This paper presents a uniform analytical model by energy method and Fourier series expansion to analyze detent force in uneven magnetic field for permanent magnet linear synchronous motor (PMLSM). The model reveals that detent force in long-primary type is mainly influenced by non-ideal distribution of permanent magnet magnetic motive force, while nounified air-gap permeance makes a great impact on detent force of short-primary type. Hence, magnetic field similarity of motor design techniques referring rotary counterpart are adopted. For long-primary type novel method of splitting edge magnets is proposed to reduce end effects force, and optimal widths of edge tooth in short-primary type also verify the effectiveness of magnetic field similarity. The experimental results validate finite element analysis results.
\end{abstract}

Key words: End effects, Energy method, Harmonics, Magnet shape

\section{Introduction}

Detent force in PMLSM will result in a periodic fluctuation with mover position, and it is particularly undesirable in motion control and high-precision applications. Traditionally it is deemed that the periodic detent force is composed of slot effects force and end force. In past years, many analysis models and optimization approaches for reducing detent force have been presented and discussed.

The slot effects of detent force is analogous to the cogging torque in rotary motor, so motor design considerations for two configurations are similar, such as adjusting width of PM, skewing and semi-closed slots [1]-[4] etc., However, it is well known that flux distribution is intrinsically different between linear motor and its rotary counterpart [5], so those technologies should be reconsidered.

For end effects of detent force, it is special phenomenon of linear motor resulting from finite geometry parts. Many foregoing efforts have been carried out to reduce it [6]-[11], and analytical method is single edged model. Perceiving from [6] and [7], end force can be reduced considerably by adopting a suitable stator length and chamfering. Yu-wu Zhu etal [8] present auxiliary poles design to suppress end effect of detent

\footnotetext{
* Department of Electrical Engineering, Harbin Institute of

Technology, Harbin, China(liliyi@hit.edu.cn, ccchan@eee.hku.hk ) Received 24 November 2012; Accepted 17 January 2013
}

force. In fact, all of aforementioned researches show that improvement of end effects in linear motors will be beneficial to reduce detent force considerably.

In this paper we adopt the concept of magnetic field similarity, and its goal is to reform even magnetic field in linear motor referring rotary one by motor structure design techniques. Firstly, a uniform analytical model is established by energy method and Fourier series expansion to estimate the influence of end effects on detent force. Then, optimized technologies of magnetic field similarity for long- and short-primary type are discussed respectively. The effectiveness of proposed method is verified by finite element analysis and testing.

\section{Detent Force Analysis by Energy Method}

\subsection{Magnetic Field in PM Linear Motor}

Because of open longitudinal ends, magnetic field distribution of a PMLSM is different from rotary counterparts. Generally PMLSM is classified into longprimary type and short-primary type [12]. Based on identical parameters, flux distributions of two types of PMLSM are investigated with finite-element simulations, as shown in Fig. 1(a) and (b). The influences of end effects on airgap flux distribution in two configurations are different. Hence, optimized technologies need to be discussed respectively. 


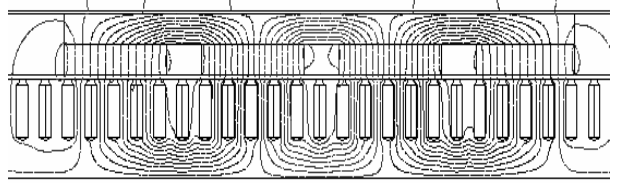

(a)

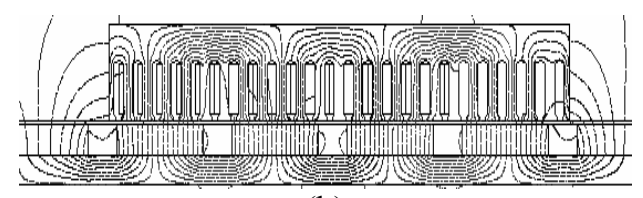

(b)

Fig. 1. Flux line distribution. (a) Long-primary type PMLSM. (b) Short-primary type PMLSM.

In this paper a uniform analytical model based on energy method and Fourier series expansion are built to evaluate influence of uneven flux distribution on detent force. Though energy method is well known for cogging force analysis in rotary motor [1], few researchers adopt this model for detent force analysis in linear motor.

\subsection{Energy Method under Ideal Condition}

On the assumption that ideal magnetic circuit of PMLSM is shown in Fig.2, it is described that length of primary $L_{p}$ and secondary $L_{s}$ are infinite $(2 p \tau, p$ is $\infty)$, so there is no end effects, and linear motor is regarded as portion of an rotary machine. A simple airgap permeance function $G^{2}(x)$ and quadratic PM magnetic motive force $(\mathrm{MMF})\left[A\left(x, x_{0}\right)\right]^{2}$ can be expressed by Fourier series as (1),(2).

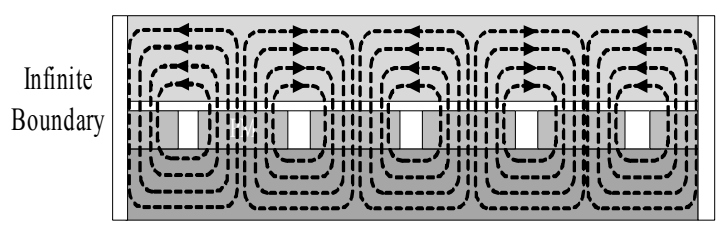

Fig. 2. Ideal model of PMLSM.

$$
\begin{gathered}
G^{2}(x)=G_{0}+\sum_{\mu}\left(G_{\mu a} \cos \mu k x+G_{\mu b} \sin \mu k x\right) \\
{\left[A\left(x, x_{0}\right)\right]^{2}=A_{0}+\sum_{v}\left[A_{v a} \cos v k\left(x+x_{0}\right)+A_{v b} \sin v k\left(x+x_{0}\right)\right]}
\end{gathered}
$$

where, $x_{0}$ is at centerline of one tooth, which is defined as zero position. $x$ is mover [11]position and $k=\pi / \tau . \mu$ and $v$ are respectively harmonic order in length of $2 p \tau$.

Compared with energy variation in air gap, that in the iron is usually negligible [8], so magnetic energy of air gap is given by

$$
\begin{aligned}
W & =\frac{1}{2 \mu_{0}} \int_{v} B^{2}\left(x, x_{0}\right) \mathrm{dV} \\
& =\frac{1}{2 \mu_{0}} \int_{0}^{L_{e f}} \int_{0}^{2 \pi}\left[A\left(k x, k x_{0}\right)\right]^{2} G^{2}(k x) \cdot k d \theta d z
\end{aligned}
$$

Based on energy method, detent force is obtained from energy variation.

$$
F_{d e n}(x)=\frac{\partial W(x)}{\partial x}
$$

Each harmonic component can be simplified as

$$
F_{d e n}(x)=\left\{\begin{array}{c}
0, \mu \neq v \\
\frac{\pi^{2} L_{e f}}{2 \mu_{0} \tau} v\left[\begin{array}{l}
\left(A_{v a} G_{v a}+A_{\mu b} G_{\mu b}\right) \sin v k x+ \\
\left(A_{v a} G_{\mu b}-A_{v b} G_{\mu a} \cos v k x\right)
\end{array}\right], \quad \mu=v
\end{array}\right.
$$

Under ideal condition, period of airgap permeance function $G^{2}(x)$ is slot pitch $\tau_{s}$, and period of $\left[A\left(x, x_{0}\right)\right]^{2}$ is pole pitch $\tau$, so harmonic order $\mu$ and $v$ in displacement $2 p \tau$ are given by

$$
\left\{\begin{array}{c}
\mu=m / \tau_{s}, m=1,2,3 \ldots \\
\nu=n / \tau, n=1,2,3 \ldots
\end{array}\right.
$$

When $\mu=v$, that is $m / \tau_{s}=n / \tau$, detent force can be acquired by superposition of two groups of harmonic components. Considering symmetry and periodicity, sinusoidal coefficient of $G^{2}(x)$ and $\left[A\left(x, x_{0}\right)\right]^{2}$ is zero. The resultant detent force is, therefore, rewritten as

$$
F_{d e n}(x)=\left\{\begin{array}{c}
0, \mu \neq v \\
\frac{\pi^{2} L_{e f}}{2 \mu_{0} \tau} v\left(A_{v a} G_{\mu a} \sin \mu x\right), \mu=v
\end{array}\right.
$$

It is well-known that $m Z=n 2 p$, goodness[12] factor $C_{T}$ which is proportional to the product of slot number $N_{L}$ and pole number $N_{p}$ is inversely proportional to the least common multiple between $N_{L}$ and $N_{p}$. Therefore, the higher the factor $C_{T}$, the larger the cogging torque [13].

However, in linear motor primary and PM secondary can not be matched, so ideal model is nonexistent and finite length parts exerts magnetic field distribution.

\subsection{Uneven Distributed of $\left[A\left(x, x_{0}\right)\right]^{2}$}

If $L_{p}>>L_{s}$, MMF distribution of each pole becomes asymmetric because of interrupted magnetic path of outmost magnets as shown in Fig. 3. 


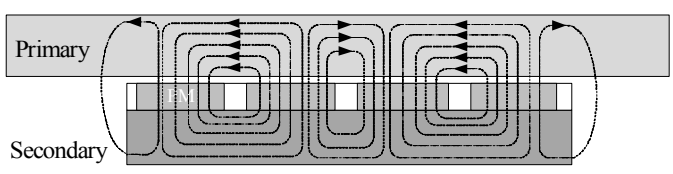

Fig. 3. The long-primary type

Here, $L_{p}=\infty$, so $G_{\mu b}=0$. Therefore, according to (5), detent force can be obtained as

$$
F_{d e n}(x)=\frac{\pi^{2} L_{e f}}{2 \mu_{0} \tau} \nu G_{\mu a}\left(A_{v a} \sin v k x-A_{v b} \cos v k x\right)
$$

Compared with ideal condition, period of $\left[A\left(x, x_{0}\right)\right]^{2}$ is $2 p \tau$, then

$$
m / \tau_{s}=n / 2 p \tau
$$

Accordingly, $m Z=n$, so harmonics order of detent force is $Z$ and its multiples. Besides, the fundamental order fluctuates in periods of $\tau_{s}$ in long-primary type. It is noted that detent force in long-primary type differs from traditional "cogging force", which occurs in uneven air gap magnetic field, so the goodness factor $C_{T}$ is not applicable.

\subsection{Nonunified Distributed of $G^{2}(x)$}

If $L_{s}>>L_{p}$, finite primary has a great influence on harmonic components of $G^{2}(x)$, as illustrated in Fig. 4. When it interacts with even distributed of $\left[A\left(x, x_{0}\right)\right]^{2}$, here, $A_{\mu b}=0$, detent force can be rewritten as

$$
F_{d e n}(x)=\frac{\pi^{2} L_{e f}}{2 \mu_{0} \tau} v A_{v a}\left(G_{\mu a} \sin \mu k x+G_{\mu b} \cos \mu k x\right)
$$

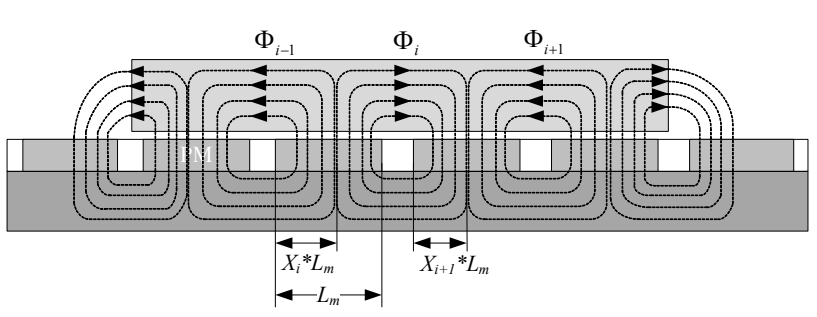

Fig. 4. The short-primary type.

Similarly, period of nonunified distributed of $G^{2}(x)$ is $2 p \tau$, hence, $m / Z \tau_{s}=n / \tau$, that is

$$
m=n 2 p
$$

For short-primary type, the harmonic order of detent force is $2 p$ and its multiple. Besides, the fundamental order harmonic fluctuates in periods of $\tau$. In order to evaluate uneven flux distribution in following analysis, reluctance model presented in [5] are adopted. The free parameter $x_{i}$ can take any value between 0 and 1 .

For rotary machine, fractional slot technology is to increase frequency of cogging force and reduce its amplitude for minimization, whereas PMLSM has fixed frequency of detent force with only variation of $N_{L}$ or $N_{p}$.

\subsection{Analysis Model and Its Specification}

The PMLSM prototype of four poles is moving magnet type and its parameters are listed in Table I. The main parameters in short-primary type are identical with long-primary type, and PM secondary is extended.

The field calculation of detent force is based on twodimensional finite-element formulation as follows.

$$
F_{\text {den }}(x)=\frac{d W(x)}{d x}=\frac{\partial}{\partial x}\left[\int v_{v}\left(\int_{0}^{H} B d H\right) d V\right]
$$

\section{Detent Force In Long-Primary Type}

From above model, we can see that detent force of long-primary type is mainly influenced by non-ideal distribution $\left[A\left(x, x_{0}\right)\right]^{2}$. Hence, the key parts of magnetic field similarity are focused on PMs secondary. As indicated by [5], longitudinal end effects stem from disturbance of coupled flux of outmost magnets, and change of flux pattern propagates in wards in motor. Hence, we present splitting edge magnets method to improve pairwise coupled flux pattern.

Table 1. The specifications of prototype

\begin{tabular}{|c|c|c|}
\hline Symbol & Item & Value \\
\hline$\tau$ & pole pitch & $0.06 \mathrm{~m}$ \\
\hline$\tau_{s}$ & slot pitch & $0.01 \mathrm{~m}$ \\
\hline$W_{s}$ & width of slot & $0.005 \mathrm{~m}$ \\
\hline$h_{s}$ & depth of slot & $0.022 \mathrm{~m}$ \\
\hline$I_{m}$ & rated current & $2.5 \mathrm{~A}$ \\
\hline$q$ & slot/pole/phase & 2 \\
\hline$L_{M}$ & width of PM & $0.045 \mathrm{~m}$ \\
\hline$h_{m}$ & thickness of PM & $0.006 \mathrm{~m}$ \\
\hline$B_{r}$ & residual flux density & $1.2 \mathrm{~T}$ \\
\hline$g$ & length of Air gap & $0.001 \mathrm{~m}$ \\
\hline & material & Nd-Fe-B \\
\hline
\end{tabular}

\subsection{Splitting Edge Magnets Method}

From FEM results shown in Fig. 5, we discover that the bigger harmonics for long-primary type is relatively dispersed to $Z, 2 Z, 3 Z$, etc. We divide edges magnets into two same pieces, and the FFT results indicate that detent force of splitting edge magnets model is less than that of 
conventional even pole magnets, and saturation of iron core and yoke induced by end effects is also relieved.

\subsection{Design Criteria and optimized results}

For PMLSM with splitting edge magnets, coupled flux at longitudinal ends transform into more evenly distributed flux away from ends, so the optimal width of PM need to be adjusted shown in Fig. 6.

Fig. 6 shows variation of detent force according to width of PM. Furthermore, the results in Fig. 7 indicate that adopting this method end flux leakage is reduced, so electromagnetic force is enhanced.

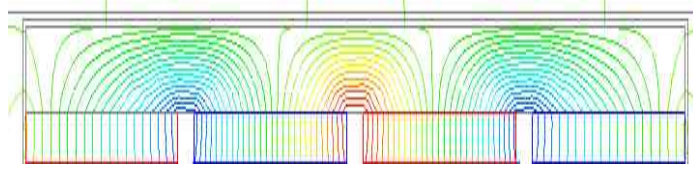

(a)

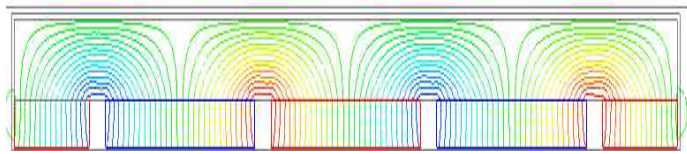

(b)

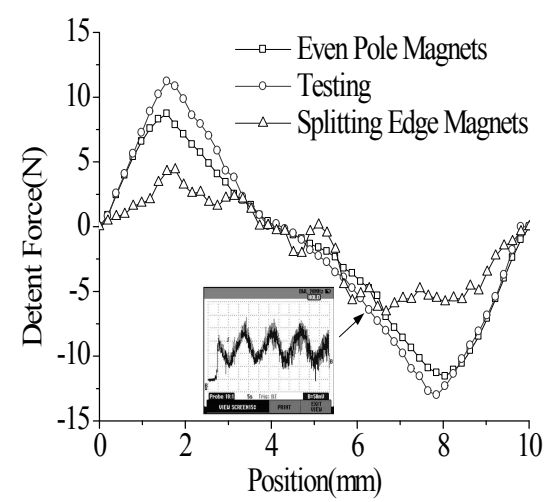

(c)

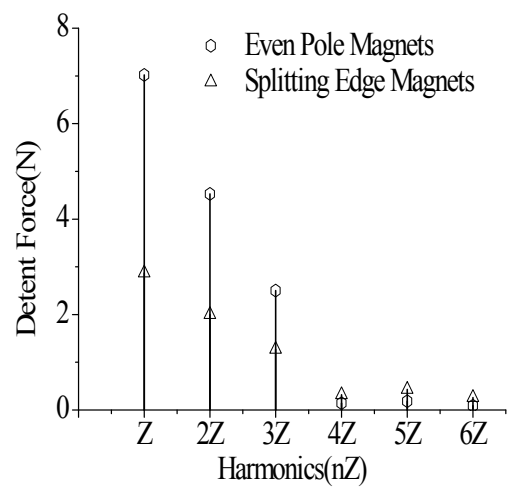

(d)

Fig. 5. Optimization results. (a) Magnetic field of even pole magnets. (b) Magnetic field of splitting edge magnets. (c) Detent force wave in different PM configurations. (d) Harmonics analysis.

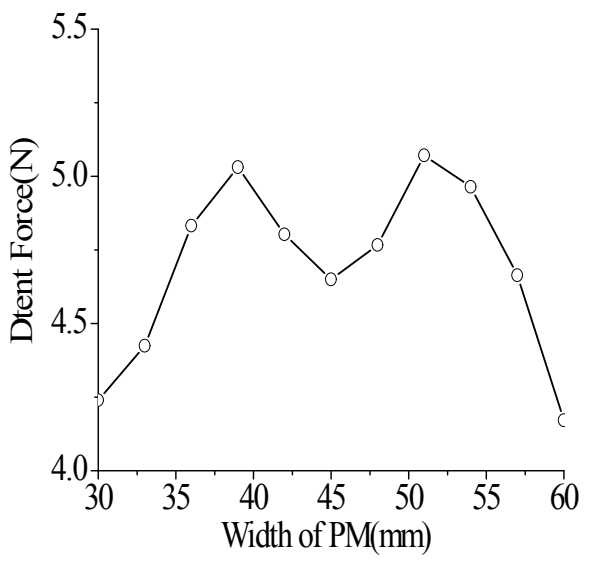

Fig. 6. Detent force $v s$ width of PM.

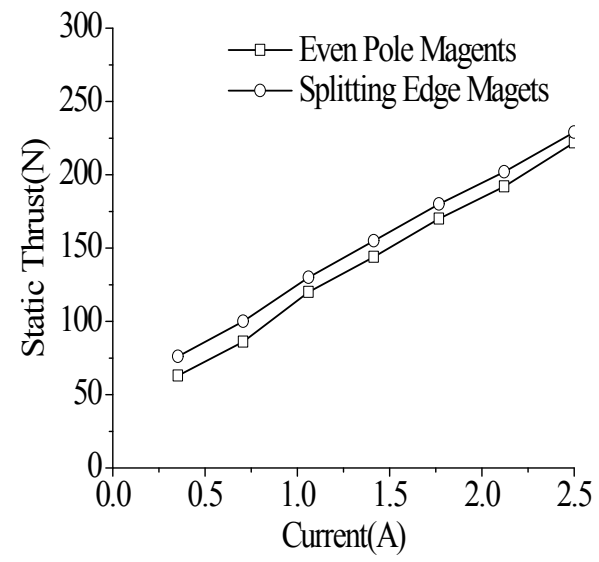

Fig. 7. Static thrust with different PM configurations.

\subsection{Arc Shape PM}

In practical applications, method of arc shape PM designs is usually presented to eliminate high order harmonic of $\left[A\left(x, x_{0}\right)\right]^{2}$. In our laboratory we manufacture two kinds of mover as shown in Fig. 8 (a) and (b). As indicated by Fig. 8(d), high order harmonics of detent force such as $2 Z$ and $3 Z$ can be eliminated markedly, but fundamental component are still higher.

\subsection{Comparison and Discussion}

The positive effects of splitting edge magnets method for long-primary type are evident. But this method is more suitable when linear motor has just a few PM poles. While the method of arc shape PM designs is applicable extensively,but it has greater difficulties in manufacturing relative to splitting edge magnets method.

\section{Detent Force In Short-Primary Type}

In short-primary type many methods have been proposed to bring detent force down. In essence, the aim of these technologies is to adjusting end permeance $G^{2}(x)$. 
This paper takes shape optimization of edge tooth for example, and analysis results of magnetic field similarity are explained by reluctance model in [5].

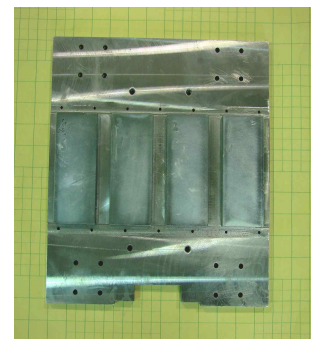

(a)

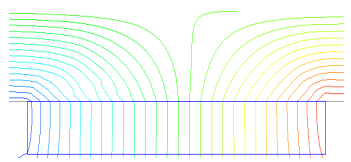

(c)

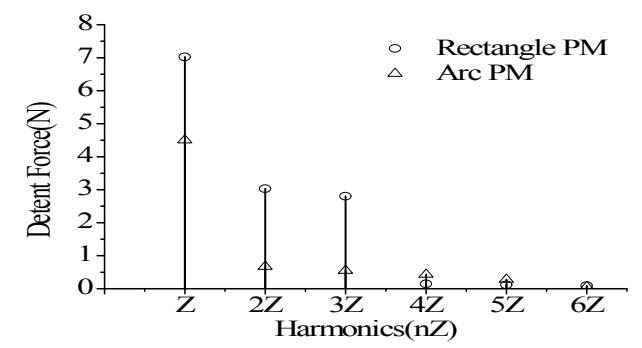

(d)

Fig. 8. Optimization results. (a) Retangle PM. (b) Arc PM. (c) Magnetic field in different magnet shape. (d) Harmonics analysis.

\subsection{Harmonic analysis}

Based on the results of Fig. 9, we discover that the stronger harmonic in short-primary type is fundamental component, and influence of nonideal distributed of $G^{2}(x)$ is exceedingly large relative to long-primary type.

\subsection{Optimization of Edge Tooth}

Based on reluctance model in Fig. 4, magnetic energy associated with every flux path can be acquired as

$$
\mathrm{E}=\sum_{i=1}^{N-1} K\left(\frac{\Phi_{i}^{2}}{1-x_{i}}+\frac{\Phi_{i}^{2}}{x_{i+1}}\right)
$$

where, $x_{i}$ is defined as flux distribution coefficient. The coefficient $K$ is involved with structural parameters, such as the stack length $L_{e f}$, aigap length $g$, thickness of magnet $h_{m}$ i.e, and it can be simply expressed as

$$
K=\frac{h_{m}+g}{2 \mu L_{e f} L_{m}}
$$

So detent force is obtained as

$$
\mathrm{F}_{\mathrm{den}}=\frac{\partial E}{\partial \mathrm{x}}=\sum_{i=1}^{N-1} K \frac{\partial}{\partial x}\left(\frac{\Phi_{i}^{2}}{1-x_{i}}+\frac{\Phi_{i}^{2}}{x_{i+1}}\right)
$$

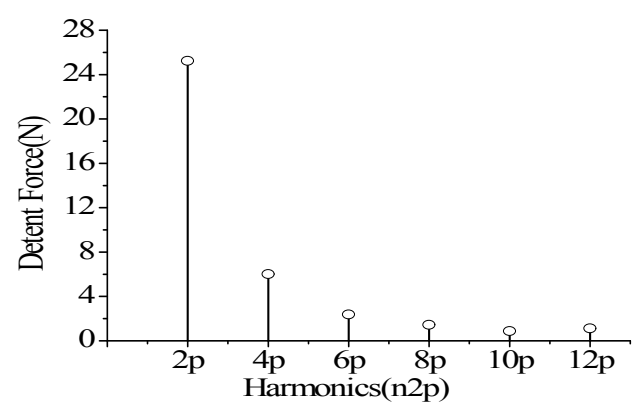

Fig. 9. Harmonics analysis.

Fig. 10 shows the variation of detent force and end magnetic field with different width of edge tooth. Comparing harmonics of model I and model II in Fig. 10 (d), it is clear that fundamental component of model II decreased markedly relative to that of model I.

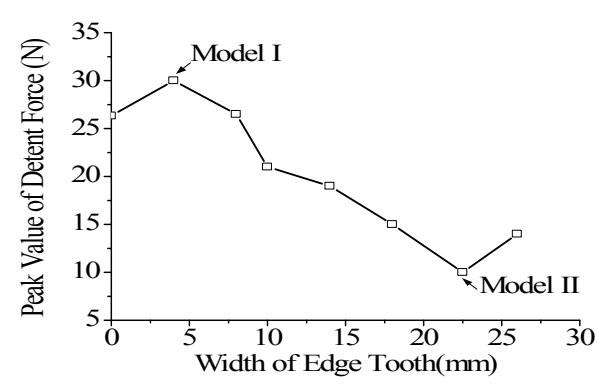

(a)

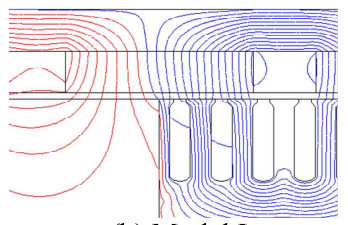

(b) Model I

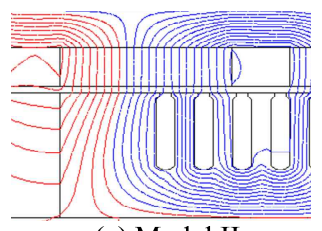

(c) Model II

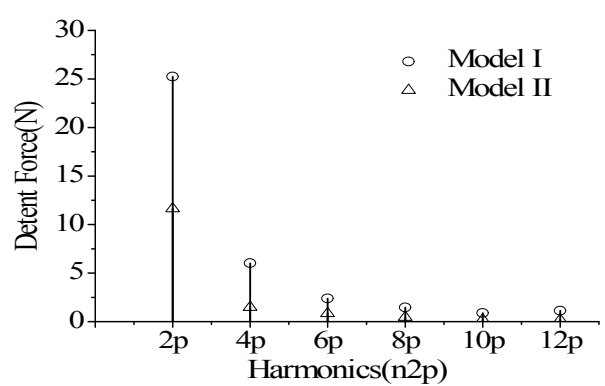

(d)

Fig.10. Optimization results. (a) Peak value of detent force with width of edge tooth. (b) Magnetic field in model I. (c) Magnetic field in model II. (d) Harmonics analysis. 


\subsection{Skewing}

Skewing is a well known method to minimize detent force for short-primary type, and in fact it is also effective in long-primary type. For comparison, the structural parameters are identical for two structures. Fig. 11 shows variation of thrust ripple ratio with different skew length, and the optimal skew length are different for two structures, which has same pole pitch. Moreover, we found that thrust ripple ratio decreases persistently with increase of skew length in long-primary type. However, thrust ripple of short-primary type has the lowest point, after that, skewing will have little benefit.

\subsection{Comparison and Discussion}

From the above analysis results, we can found that these methods can be used effectively to reduce detent force for short-primary type linear motor. However, after changing the width of edge tooth, the flux distribution will be distorted around edge tooth when the windings are excited, so this method has an influence on BackEMF. As for skewing, electromagnetic thrust ripple will decreases evidently when skewing length increases. Hence, in application we need to consider their weak point and benefits together to achieve the requirements of performance goals.

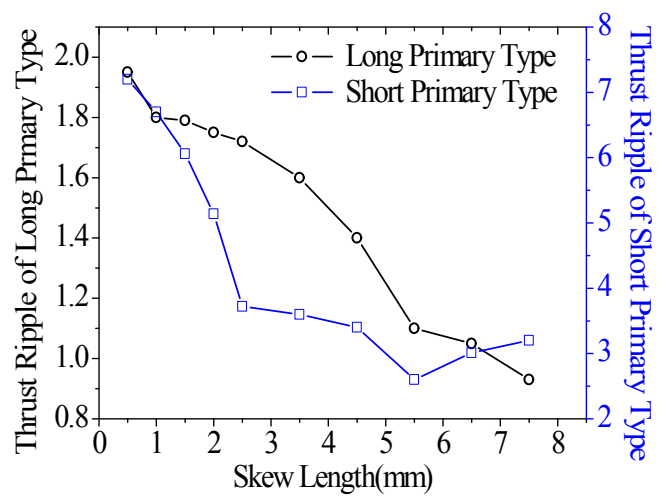

Fig. 11. Thrust ripple vs skew length.

\section{Conclusion}

In long-and short-primary type PMLSM, there are certain differences in magnet field distribution resulting from end effects. This paper has developed analytical insights by energy method and Fourier series expansion to estimate influence of end effects on detent force. The harmonics of uneven magnetic field interactions on the detent force has been clearly highlighted. For longprimary type, splitting edge magnets method can improve coupled flux pattern and is effective to enhance thrust. In short-primary type, optimization results of edge shape also verify the effectiveness of magnetic field similarity. The universal method of skewing is investigated and compared between long-primary and short-primary type. The different optimal skew length also reflects impacts of end effects on detent force.

\section{Acknowledgement}

The authors gratefully acknowledge the financial support of the National S\&T Major Project (2009ZX02207-001) and Natural Science Foundation of P. R. China (No. 51177024).

\section{References}

[1] Li Zhu, S.Z.Jiang, Z.Q.Zhu and C.C.Chan, "Analytical Methods for Minimizing Cogging Torque in Permanent Machines," IEEE Trans. Magn., vol. 45, no. 4, pp. $2023-$ 2031, Apr. 2009.

[2] Y. W. Zhu and Y. H. Cho, "Thrust Ripples Suppression of Permanent Magnet Linear Synchronous Motor," IEEE Trans. Magn., vol. 43, no.1, pp.2537-2539, Jun. 2007.

[3] In Soung Jung, Jin Hur and Dong Seok Hyun, "Performance Analysis of Skewed PM Linear Synchronous motor according to Various Design Parameters," IEEE Trans. Magn., vol. 37, no.5, pp.36533656, Sep. 2001.

[4] Nicola Bianchi, Silverio Bolognani. Design techniques for reducing the cogging torque in surface-mounted $\mathrm{PM}$ motors. IEEE Trans. Ind. Appl., vol. 38, no. 5, pp. 12591265, Sep.2002.

[5] O. Danielsson and M. Leijon, "Flux Distribution in Linear Permanent-Magnet Synchronous Machines including Longitudinal End Effects," IEEE Trans. Magn., vol. 43, no. 7, pp. 3197-3201, Jul. 2007.

[6] Masaya Inoue and Kenji Stato, "An Approach to a Suitable Stator Length for Minimizing the Detent Force of Permanent Magnet Linear Synchronous Motors," IEEE Trans. Magn.,vol. 36, no. 4, pp. 1890-1893, Jul. 2007.

[7] Yu Wu Zhu, Dae Hyun Koo and Yun Hyun Cho, "Detent Force Minimization of Permanent Magnet Linear Synchronous Motor by Means of two Different Methods," IEEE Trans. Magn., vol. 44, no. 11, pp. 4345-4348, Nov. 2008.

[8] Yu-wu Zhu, Sang-Gun Lee, Koon-Seok Chung, and YunHyun Cho, "Investigation of Auxiliary Poles Design Criteria on Reduction of End Effect of Detent Force for PMLSM,” IEEE Trans. Magn., vol. 45, no. 6, pp. 28632866, June. 2009.

[9] Martine G, Atencia J, Martine Iturralde, etal, "Reduction of Detent Force in Flat Permanent Magnet Linear Synchronous Machines by Means of Three Different Methods," Electric Machines and Drives Conference, IEMDC'03. pp.1105 - 1110, Jun. 2003

[10] Sung Whan Youn, Jong Jin Lee, Hee Sung Yoon and Chang Seop Koh, "A New Cogging-Free Permanent Magnet Linear Motor,” IEEE Trans. Magn.,vol. 44, no. 7, pp. 1785-1790, Jul. 2008. 
[11] Wang Hao, Zhang Zhijing, Liu Chengying, "Compensation Methods of Longitudinal End Effects in PermanentMagnet Linear Synchronous Motor," Proceedings of the CSEE, 2010, 30(36):46-52(in Chinese).

[12] In Soung Jung, Sang Baeck Yoon and Jang Ho Shim, etal, "Analysis of Forces in a Short Primary Type and a Short Secondary Type Permanent Magnet Linear Synchronous otor," IEEE Trans. Energy Convers.,vol. 14, no. 4, pp. 1265-1269, Dec. 1999.

[13] Nicola Bianchi, Silverio Bolognani, Michele Dai, and Giorgio Grezzani. Design Considerations for FractionalSlot Winding Configurations of Synchronous Machines. IEEE Trans. Ind. Appl., vol. 42, no. 4, pp. 997-1005, Sep.2006.

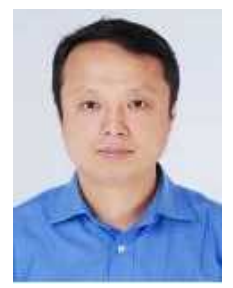

Liyi Li received Ph.D. degrees in electrical engineering from Harbin Institute of Technology, Harbin, China,in 2001. His research interests are design and control of electrical machines.

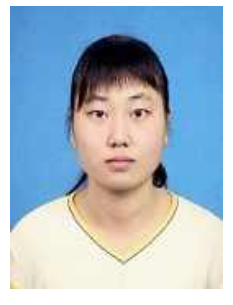

Mingna Ma received the B.E. degree in 2007 and the M.E. degree in 2009 from Harbin Institute of Technology, Harbin, China. She is currently with the Institute of Electromagnetic and Electronic Technology, Harbin Institute of Technology as a doctoral candidate from 2009. Her research areas are magnet linear synchronous motor and linear electromagnetic launcher.

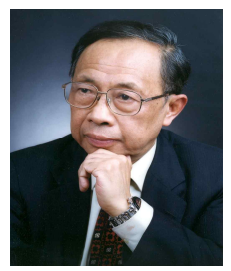

C. C. Chan received $\mathrm{BSc}, \mathrm{MSc}, \mathrm{PhD}$, HonDSc, HonDTech degrees. Honorary Professor and Former Head of the Department of Electrical and Electronic Engineering of the University of Hong Kong; Visiting Professor of MIT, University of Cambridge; Founding President of the World Electric Vehicle Association; Senior Consultant to governments and industries; Fellow of the Royal Academy of Engineering, U.K., Chinese Academy of Engineering, the Ukraine Academy of Engineering Sciences, IEEE, IET and Hong Kong Institution of Engineers. Recipient of IEE International Lecture Medal; Gold Medal of Hong Kong Institution of Engineers; "Asia's Best Technology Pioneers" by Asia week; "Father of Asian Electric Vehicles" by Magazine Global View; "Pitamaha (Grandfather) of Electric Vehicle Technology" in India; "Environmental Excellence in Transportation Award" by Society of Automotive Engineers (SAE); published 11 books, over 300 technical papers and holds 9 patents. His mission is to promote the global collaboration for the advancement of science and technology for global sustainable development. 